\title{
Advancement and Refinement of HyperSoar Modeling
}

\author{
P.H. Carter II, D.J. Pines, L. vonEggers Rudd
}

February 25, 2000

U.S. Department of Energy

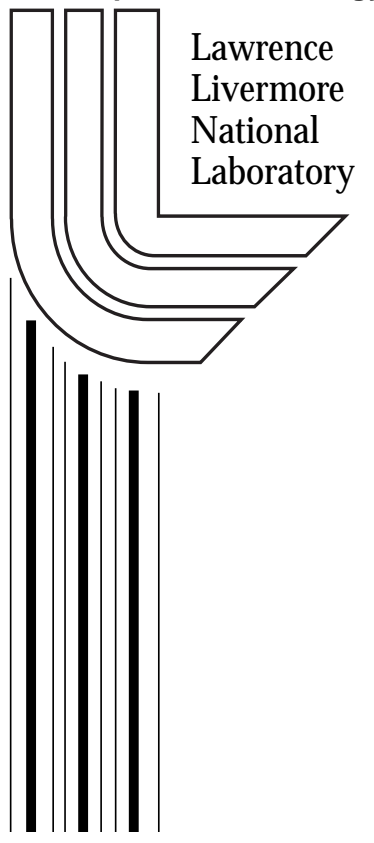




\section{DISCLAIMER}

This document was prepared as an account of work sponsored by an agency of the United States Government. Neither the United States Government nor the University of California nor any of their employees, makes any warranty, express or implied, or assumes any legal liability or responsibility for the accuracy, completeness, or usefulness of any information, apparatus, product, or process disclosed, or represents that its use would not infringe privately owned rights. Reference herein to any specific commercial product, process, or service by trade name, trademark, manufacturer, or otherwise, does not necessarily constitute or imply its endorsement, recommendation, or favoring by the United States Government or the University of California. The views and opinions of authors expressed herein do not necessarily state or reflect those of the United States Government or the University of California, and shall not be used for advertising or product endorsement purposes.

This work was performed under the auspices of the U. S. Department of Energy by the University of California, Lawrence Livermore National Laboratory under Contract No. W-7405-Eng-48.

This report has been reproduced directly from the best available copy.

Available to DOE and DOE contractors from the

Office of Scientific and Technical Information

P.O. Box 62, Oak Ridge, TN 37831

Prices available from (423) 576-8401

http://apollo.osti.gov/bridge/

Available to the public from the National Technical Information Service

U.S. Department of Commerce 5285 Port Royal Rd., Springfield, VA 22161

http://www.ntis.gov/

OR

Lawrence Livermore National Laboratory Technical Information Department's Digital Library http://www.llnl.gov/tid/Library.html 


\title{
Advancement and Refinement of HyperSoar Modeling
}

\author{
Preston Henry Carter II, Principle Investigator \\ New Technology Engineering Division, Mechanical Engineering Department, \\ Engineering Directorate \\ Lawrence Livermore National Laboratory, CA 94550 \\ Darryll J. Pines and Lael vonEggers Rudd \\ Department of Aerospace Engineering \\ University of Maryland, College Park, MD 20742
}

\begin{abstract}
This report discusses the topic of periodic cruise trajectories for hypersonic flight. An extensive review of previous work associated with periodic cruise trajectories for subsonic, supersonic and hypersonic flight is presented to provide the background for this investigation. The primary objective of this report is to discuss why periodic cruise trajectories lead to near fuel-optimal trajectories from a heuristic, mathematical and computational perspective with air breathing propulsion. Results to date indicate that periodic achieves greater fuel savings by exchanging kinetic and potential energy more efficiently. The vehicle attempts to chatter back and forth between where the vehicle wants to fly for optimum aerodynamic and propulsive performance. Results from computational simulations are inconclusive and require further work to define appropriate interfaces for aerodynamic and propulsion data decks for input into the POST software. The notional design of a vehicle to fly periodic hypersonic cruise trajectories was improved by including concepts for engine installation, flight controls and by including considerations for off-design performance. This notional design provides a better starting point for more serious and complete vehicle design studies.
\end{abstract}

\section{INTRODUCTION}

An article about HyperSoar in the October 1999 issue of "Mercedes-Benz Transport Magazine" captured the potential impact of HyperSoar to the common man:

"The idea is fascinating: fresh salmon from Alaska ready and waiting at the fishmonger's in Paris just a few hours after the catch, vital spare parts make their way from Berlin to Sydney within the space of a single afternoon, express mail from Tokyo arrives in London seven hours before take-off time. What kind of flying object could possibly move that fast?"

From investigations of our country's security needs for global reach and rapid access to orbit, we have invented a revolutionary hypersonic vehicle that is notably different from, and more efficient then, conventional aircraft. HyperSoar, (our coined name for the concept), flies at ten times the speed of sound, skipping upon the atmosphere like a rock thrown upon the surface of a lake, turning its engines on each time 
it skips upon the air and turning them off while its coasts between skips. Our project is developing the computational tools and modelling required for advancing the principles incorporated in the concept.

HyperSoar flies a Periodic Hypersonic Cruise (PHC) trajectory. The use of period control in an airplane's trajectory is a relatively new topic. The mathematical foundations of periodic control are still being constructed and the intuitive physical understanding of why these trajectories work are still being argued. Questions that arise are "Why isn't steady-state cruise optimal,"?, "What are the fundamental principles and mechanisms that make periodic trajectories work,"?, "What is the optimal trajectory,"?, etc... In part, our current work strives to provide some preliminary insights as to why PHC trajectories are fuel optimal.

Interest in periodic cruise trajectories has spanned approximately four decades since the 1950's. Initial efforts were focused on improving subsonic flight trajectories. Recent efforts have focused on hypersonic flight with a goal to carry more payload to greater distances faster and more efficiently. Such interest in hypersonic technology has spanned several new programs including, NASA'S Hyper-X and Spaceliner 100 programs and DARPA's Mach 6 missile development. While most of these new programs are focused on evaluating hypersonic technologies such as air breathing engines and thermal protection systems, vehicle designs and trajectory simulations are of key importance.

This report attempts to address one such hypersonic technology area involving periodic cruise trajectories. The discussion of periodic cruise trajectories in this report is divided into four parts:

- a chronological discussion of contributions to periodic cruise trajectories for subsonic, supersonic and hypersonic flight,

- a discussion based on fundamental physics that attempts to explain why periodic cruise achieves better fuel consumption savings over steady-state cruise,

- a mathematical analysis illustrating the sub-optimal nature of steady cruise trajectories,

- and finally, a preliminary computational investigation of periodic cruise using the software program POST.

\section{Chronological Contributions to Periodic Cruise}

It has been proven that steady-state modes of engineering processes are not always optimal and that cyclic control can produce greater efficiency. Optimal periodic control can be traced back to at least Hausen, circa 1927, for use in heat regenerators. Sanger was the first known advocate of periodic control of aerospace vehicles to increase range for an intercontinental bomber. However, recent credit has been given to Edelbaum, who in the 1950's analyzed the optimality of an energy-state model to show 
mechanisms for enhanced cruise performance by periodic cruise ${ }^{1}$. In the twenty years following Edelaum, there has been a considerable amount of effort in determining optimal periodic trajectories for subsonic and hypersonic flight.

Zagalsky, Irons, and Schultz ${ }^{2}$ showed the dilemma associated with applying the energy state approximation to the minimum fuel performance optimization problem. This dilemma was that the Maximum Principle failed to yield any solutions to the problem of maximum range cruise. This was a consequence of the fact that the velocity set was not convex. This lack of convexity allowed relaxed controllers that attained larger fuel savings compared to any control satisfying the maximum principle. Suboptimal trajectories were shown to achieve better fuel economy when an optimal control failed to exist for full-powered climbs and zero throttle glides. These sub-optimal trajectories had a minimum cruise segment and achieved fuel economies similar to the optimum relaxed controller. It was suggested that combining minimum-fuel energyclimb with classical cruises and maximum-range glides could treat the minimum-fuel problem with range constraints.

Schultz and Zagalshy ${ }^{3}$ studied three types of flight-path optimization problems for aircraft performance. These consisted of minimum fuel-fixed range, minimum time-fixed range, and minimum fuel-fixed range-fixed time. Five different types of aircraft models were evaluated using point-mass equations of motion. The maximum principle was applied to these equations and the properties of the control variables and velocity set were investigated. It was concluded that simple sets of motion equations were shown to exist which can be used with the maximum principle. These equations have the same general properties of more exact equations.

Speyer ${ }^{4}$ showed, contrary to Schultz and Zagalshy, that cruise is not a minimizing solution for minimum fuel consumption. Applying the vector control form of the generalized Legendre-Clebsch condition did this. While the cruise condition had previously been found to be a doubly singular arc in the calculus of variations, the singular arc was shown to not be minimizing when second order necessary conditions were taken into account. The aircraft model used had thrust and flight path angle as the control variables. The result was consistent with the energy state approximation and while constant mass was assumed in the analysis the result would be the same for a changing mass.

Shultz ${ }^{5}$ responded to Speyer's work by using a higher order set of equations to show the fuel optimality of cruise. In this model lift and thrust were the controls. It was shown that the necessary condition for optimization including the generalized LegendreClebsch condition is satisfied at the cruise condition. This allowed partial throttle cruises to be a solution for the minimum fuel-fixed range problem. An explanation for why this case worked is that in the energy state equation, fast changes in altitude can occur without

\footnotetext{
Edelbaum, T., "Maximum Range Flight Paths," United Aircraft Corp., Rept. R-22465-24, 1955

Zagalsky, N. R., Jr., R. P. I., and Schultz, R. L., "Energy State Approximation and minimum-Fuel Fixed Range Trajectories," Journal of Aircraft, Vol. 8, No.6, 1971, pp. 488-490.

3 Schultz, R., L. and Zagalshy, N. R., "Aircraft Performance Optimization,” Journal of Aircraft, Vol. 9, No. 2, 1972, pp. 108-114.

4 Speyer, J. L., "On the Fuel optimality of Cruise," Journal of Aircraft, Vol. 10, No. 12, 1973, pp. $763-$ 765.

$5 \quad$ Schultz, Rl L., "Fuel Optimality of Cruise,” Journal of Aircraft, Vol. 11, No. 9, 1974, pp. 586-587.
} 
a change in induced drag, while in the case of lift-thrust control large changes in altitude require large changes in lift forces and thus large changes in induced drag.

Gilbert $^{6}$ was able to show that time-dependent periodic control can improve the fuel economy of cruise vehicles. Four time-dependent controls were analyzed. These consisted of a relaxed steady-state (RSS) control, a quasi-steady-state (QSS) control, a quasi-relaxed-steady-state (QRSS) control, and a normal steady-state control. It was shown with examples that QRSS control could yield better fuel performance over the other three types of control. This work derived properties of optimal cost functions that were dependent on the minimum required average speed. The ability of improved fuel savings through the use of RSS, QSS, and QRSS control was evaluated in terms of the assumptions on vehicle drag and fuel consumption functions. An interesting example of a two-engine gas turbine ship was described in the conclusions section.

Gilbert and Parsons ${ }^{7}$ treated the optimality of cruise as an optimal periodic control problem, which relied on extensive theoretical development when similar questions of steady-state solutions were studied in the field of chemical engineering in the 1960's. Only a relaxed steady-state (RSS) analysis was used and applied to an energy state model. A periodic control problem corresponding to the energy-state approximation was used. An F-4 example was used for numerical calculations in which a four-segment cycle was used as an approximation to the optimum RSS cruise. The four segments corresponded to a zero-thrust glide, a dive, a maximum thrust climb, and a zero-thrust climb. The results showed a $1.7 \%$ savings in the RSS fuel consumption over the optimal classical cruise. The improvement became greater when altitude constraints were used. A more general idealized aircraft model was used to show that greater potential fuel savings could be realized for high thrust to drag, low wing loading aircraft.

Speyer $^{8}$ expanded on previous work by conducting a frequency-domain analysis of the cruise arc. A more stringent second-order analysis of optimality using the Goh transformation was used. The results proved that the cruise arc was not optimal. Numerical results were produced using oscillatory control, suggested by the second variation problem in the frequency domain. The results showed a smaller fuel cost than the cruise arc. Two physical insights as to why cyclic operation improves fuel were given: 1) Fuel efficiency is improved at constant energy by chattering between where the aircraft is aerodynamically efficient and power efficient with respect to thrust. 2) Dynamic considerations only cause the non-optimality. If either the maximum L/D is low or the velocity is high the frequency analysis shows that a cyclic process will be minimizing, not the steady-state cruise. This work made no attempt to find an optimal arc solution.

Houlihan, Cliff, and Kelly ${ }^{9}$, using a similar approach as Glibert and Parsons, studied chattering cruise. The work used an idealization of time-shared operation

6 Gilbert, E. G., "Vehicle Cruise: Improved Fuel Economy by Periodic Control," Automatica, Vol. 12, 1976, pp. 159-166.

7 Gilbert, E. G. and Parsons, M.G., "Periodic Control and the Optimality of Aircraft Cruise," Journal of Aircraft, Vol. 13, No. 10, 1976, pp. 828-830.

$8 \quad$ Speyer, J. L., "Nonoptimality of the Steady-State Cruise," AIAA Journal, Vol. 14, No. 11, 1976, pp. 1604-1610.

9 Houlihan, S.C., Cliff, E. M., and Kelley, H. J., "Study of Chattering Cruise," Journal of Aircraft, Vol. 19, No. 2, 1982, pp. 119-124. 
between two Mach number/altitude points. A hodograph figure was given which showed a characteristic non-convexity, leading to chattering. This graph was analyzed and the authors made attempts to relate this non-convexity to aerodynamic and propulsion parameters. Two examples were given along these lines. The first was a simple model of a vehicle with altitude and Mach number independence. The second was a computational study of several aircraft with altitude dependence and both Mach number dependence and independence. An examination of specific-energy dependence of the hodograph figure's shape was then conducted. It was shown that low energy ranges produce substantial improvements but that the best improvement found in the cruise energy range was about $5 \%$.

Speyer, Dannemiller, and Walker ${ }^{10}$ analyzed the periodic optimal cruise of an atmospheric vehicle. This work applied the second-order conditions for local optimality to a vehicle operating in the hypersonic flight regime. The closed periodic path was numerically determined using a shooting method. A solution for a flat Earth was found to be a local minimum, yielding a fuel usage savings of $4.2 \%$ over the steady-state cruise solution. An attempt was done to use these techniques for a spherical Earth but with no success. However, the spherical Earth solutions still showed increased savings in fuel usage over the corresponding steady-state solutions.

Sachs and Christodoulou ${ }^{11}$ analyzed and compared an optimal periodic control problem for maximum endurance to that of steady-state endurance. The minimum principle was applied with controls being lift coefficient and throttle. An altitude constraint was imposed for the optimal solution. A basic characteristic of endurance flight with optimal cyclic control was shown with the important effect of specific energy added to the aircraft per fuel consumed. Two flight regions were shown to exist. The first is a maximum thrust, while the second is a minimum thrust where the energy added to in the first region is used to gain as much endurance as possible. Power plant effects were also shown to affect the ability of periodic control to improve endurance. Altitude effects were also discussed and it was concluded that cyclic endurance yields best values within the flight envelopes of the aircraft considered. With these considerations, endurance improvements of $63.5 \%$ could be realized.

Grimm, Well, and Oberle ${ }^{12}$ have treated the minimization of fuel consumption as an optimal periodic control problem with periodic boundary conditions. Their work was subdivided into two problems. The fist problem was with constant weight while the second allowed a changing weight. Numerical solutions were accomplished with a multiple shooting method that consisted of bang-bang control actions for power settings. A typical optimal control strategy was used and the solutions satisfied the second-order sufficiency condition for a weak local optimum. An F-4 model aircraft was used and a fuel savings of approximately $2 \%$ was found. The non-periodic changing weight solution appeared to be similar to the periodic constant mass solution and the difference between a constant and variable weight was said to be a primarily theoretical nature.

10 Speyer, J. L., Dannemiller, D., and Walker, D., "Periodic Optimal Cruise of an Atmospheric Vehicle," Journal of Guidance, Vol. 8, No. 1, 1985, pp. 31-38.

11 Sachs, G. and Christodoulou, T., "Endurance Increase by Cyclic Control," Journal of Guidance, Vol. 1, No. 9 1986, pp. 616-622.

12 Grimm, W., Well K. H., Oberle, H. J., "Periodic Control for Minimum-Fuel Aircraft Trajectories," Journal of Guidance, Vol. 9, No. 2, 1986, pp. 169-174. 
Chuang and Speyer ${ }^{13}$ determined fuel optimal periodic cruise paths for a hypersonic vehicle with realistic aerodynamic and scram-jet models. A minimizingboundary-condition method, which is a modification of the shooting method, was used to increase the region of convergence of the solution to the low-point boundary value problem. The resulting first-order sufficient extremal trajectories were shown to be locally minimizing by a second-order sufficient extremal trajectories were shown to be locally minimizing by a second-order sufficiency condition. With the engine-off drag penalties, little improvement was obtained over the steady-state cruise. Without this penalty, fuel savings of $5 \%$ were realized.

Sachs and Christodoulou ${ }^{14}$ continued their work by formulating an optimal cyclic control problem for fuel consumption of subsonic aircraft in range and endurance flight. For cruise, the incompressible and compressible flight regimes were treated separately because each of them showed specific effects for optimal cyclic cruise. Vehicle dynamics and aerodynamics were similar to previous works. Necessary conditions for optimality were determined by applying the minimum principle. Altitude constraints were allowed for both cases of touching the altitude boundary at a point and staying on the boundary for a finite time. For optimal cyclic cruise in the incompressible flight regime, thrust was seen as a major factor with higher thrust levels resulting in larger amplitudes of the changes of speed and altitude, showing a more pronounced oscillatory behavior. Results also showed that gains could be significantly increased when more thrust is available. The effects of compressibility acted like a barrier with maximum changes in the flight behavior being reduced. Endurance flight was improved by cyclic control then range cruise with thrust, again, being a significant factor.

Wany and Speyer $^{15}$ developed the necessary and sufficient conditions for a periodic path to be a weak local minimum. The problem was developed from the second variation about the extremal path that had a free period. The conditions were established through a matrix inequality and the existence of a solution to the Riccatti differential equation over the period. The work was extended to get the optimality conditions for infinitely repeated periodic processes. It was shown that a necessary condition for optimality was a real symmetric periodic solution to the Ricatti differential equation. Sufficiency for optimality required these criteria along with requirements on the eigenvalues of the monodromy matrix.

Wang, Speyer, and Dewell ${ }^{16}$ developed a feedback controller to implement optimal periodic processes to a physical system. This controller was named a periodic regulator and allows state deviations and constant parameter variation. The periodic regulator brings the perturbed plant back to a neighboring optimal periodic path. This is done so that the infinite-time second-variation of the cost is minimized. This periodic regulator was shown to have simpler properties than other periodic regulators since the

13 Chuang, C. H. and Speyer, J.L., "Periodic Optimal Hypersonic Scramjet Cruise," Optimal control Applications and Methods, Vol. 8, 1987, pp. 231-242.

14 Saxhs, G. and christodoulou, T., "Reducing Fuel Consumption of Subsonic Aircraft by optimal Cyclic Cruise," Journal of Aircraft, Vol. 24, No. 9, 1987, pp.616-622.

15 Wang, Q. and Speyer, J. L., "Necessary and Sufficient Conditions for Local Optimality of a Periodi c Process," SIAM Journal on Control and Optimization, Vol. 28, No. 2, 1990, pp. 482-497.

16 Wang, Q., Speyer, J. L., and Dewell, L. D., "Regulators for Optimal Periodic Processes," IEEE Transactions on Automatic Control, Vol. 40, No. 10, 1995, pp. 1767-1778. 
periodic path was optimal with respect to a time-averaged cost. The periodic regulator was applied to a simple two-state periodic optimal control problem with demonstrated its convergence properties.

Speyer ${ }^{17}$ wrote a summary of all his and his students pioneering work over the past twenty-five years in optimal periodic control for aircraft cruise. While he states that he did not intend the work to be a survey of the field, it is the most complete review of the subject to date with references of most of the important work by various authors. Reviews of the optimal periodic control problem, numerical computations of periodic optimal cruise with applications to hypersonic vehicles, and regulators for optimal periodic processes were covered.

Recently Chuang and Morimoto ${ }^{18}$ have analyzed optimal cruise trajectories for a hypersonic vehicle with constraints. Hypersonic periodic trajectories were optimized using a sub-optimal initial guess that parameterized the altitude profile. This initial guess was then used in the minimization-boundary-condition optimization. Heating rates and load factors were used as constraints in the optimization. The vehicle model was constructed using numerical data and figures from available space plane literature. The unconstrained optimal solution showed an $8.12 \%$ fuel savings over steady state cruise. With a heating rate constraint of $400 \mathrm{~W} / \mathrm{cm}^{2}$, the fuel savings reduced to $2.45 \%$. With just a load factor constraint of $7 \mathrm{~g}$ 's, the fuel savings did not change much from the unconstrained solution. An optimal solution with both a heating rate constraint of 1158 $\mathrm{W} / \mathrm{cm}^{2}$ and a load factor constraint of $7 \mathrm{~g}$ 's produced a fuel savings of $8.09 \%$.

Carter, Pines, and Rudd ${ }^{19}$ have extended the original work of Sanger/Eggers to provide a design level analysis of approximate performance of periodic hypersonic cruise when compared to other trajectories typically considered (Sub-orbital, boost-glide, steady-state cruise) for global reach applications. It was shown that superior payload mass fraction ratios exist for periodic trajectories. Fig 1 shows a summary plot of this analysis.

17 Speyer, J. L., "Periodic Optimal Flight," Journal of Guidance, Control and Dynamics, Vol. 19, No. 4, 1996, pp. 745-753.

18 Chuang, C. H. and Morimoto, H., "Periodic Optimal Cruise for a Hypersonic Vehicle with Constraints," Journal of Spacecraft and Rockets, Vol. 34, No. 2, 1997, pp. 165-171.

19 Carter, P. H., Pines, D. J., and vE. Rudd, L., “Approximate Performance of Periodic Hypersonic Cruise Trajectories for Global Reach,” Journal of Aircraft, Vol. 35, No. 6, nov-Dec 1998. 


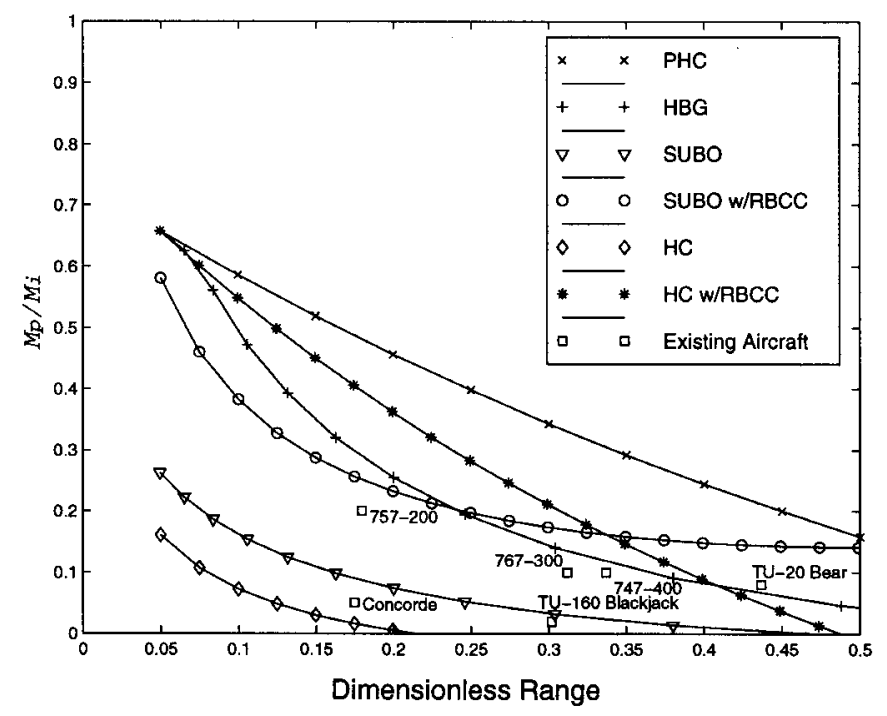

Figure 1: Comparison of payload Carrying Capability of Vehicles Flying Various Trajectory forms

Rudd, Pines and Carter ${ }^{20}{ }^{21}$ have also used the sub optimal solution method derived by Chuang and morimoto to analyze the effects of basic fundamental physics of periodic cruise trajectories and damped periodic cruise trajectories.

Some of the more notable contributions in the study of periodic cruise are summarized in Table 1, which chronologically lists researchers who have studied periodic cruise over the past 30 years. Although this table does not provide specific details regarding contributions made by these researchers, it does illustrate how a better understanding of periodic cruise trajectories has lead to a steady improvement in fuelconsumption rate savings over steady-state cruise. These steady improvements over time are primarily a result of three factors:

- Theoretical advances in the optimality of periodic cruise (application of calculus of variations to necessary and sufficient conditions),

- Better understanding of integrated vehicle flight dynamics, propulsion systems and aerodynamics for subsonic and hypersonic flight,

- Improved optimization tools for solving unconstrained and constrained two-point boundary problems (TPBVP).

\begin{tabular}{c|c|c|c|c|c|c}
\hline \hline Name & Year & Regime & Method & Optimization & Model & $\%$ Fuel Savings \\
\hline Edelbaum & 1955 & Subsonic & Th & Un & $*$ & $*$ \\
Zagalsky et al. & 1971 & Subsonic & Th & Uncon & Generic & $*$ \\
Schultz et al. & 1972 & Subsonic & Th & Un & Various & $*$
\end{tabular}

20 VE. Rudd, L., Pines, D. J., and Carter, P. H., "Sub-Optimal Damped Periodic Cruise Trajectories for Hypersonic Flight," accepted to Journal of Aircraft, 1998.

21 VE. Rudd, L., Pines, D. J., and Carter, P. H., "Improved Performance of Sub-Optimal Periodic Hypersonic Cruise Trajectories for Long-Range," AIAA $8^{\text {th }}$ International Space Planes and Hypersonic Systems and Technologies Conference, April 1998. 


\begin{tabular}{|c|c|c|c|c|c|c|}
\hline Speyer et al. ${ }^{4}$ & 1973 & Subsonic & Th & Uncon & Generic & $*$ \\
\hline Schultz ${ }^{5}$ & 1974 & Subsonic & Th & Uncon & Generic & $*$ \\
\hline Gilbert $^{6}$ & 1976 & Subsonic & Th & $*$ & $*$ & $*$ \\
\hline Gilbert et al. ${ }^{?}$ & 1976 & Subsonic & Th / Comp & Uncon & F-4 / Ideal & 1.7 \\
\hline Speyer $^{8}$ & 1976 & Subsonic & Th / Comp & Uncon & Generic & $*$ \\
\hline Houlihan et al. ${ }^{9}$ & 1982 & Subsonic & Th / Comp & Uncon & $\begin{array}{l}\text { F-4E, RPV, } \\
\text { Super cruiser }\end{array}$ & 5.9 \\
\hline Speyer et al. ${ }^{10}$ & 1985 & Hypersonic & Th / Comp & Uncon & Generic & 4.2 \\
\hline Grimm et al. ${ }^{12}$ & 1986 & Subsonic & Comp & Con & F-4 & 2.0 \\
\hline Chuang et al. ${ }^{13}$ & 1987 & Hypersonic & Th / Comp & Uncon & Space plane & 5.0 \\
\hline Sachs et al. ${ }^{14}$ & 1987 & Subsonic & Th & Con & $*$ & $*$ \\
\hline Speyer ${ }^{17}$ & 1996 & $*$ & $*$ & $*$ & $*$ & Summary \\
\hline Chuang et al. ${ }^{18}$ & 1997 & Hypersonic & Th / Comp & Uncon / Con & Space plane & 8.1 \\
\hline Carter et al..$^{19}$ & 1998 & Hypersonic & Approx. & N/A & Generic & $*$ \\
\hline Rudd et al. ${ }^{20}$ & 1999 & Hypersonic & Th / Comp & Con & Space plane & $451^{\text {st }}$ period \\
\hline
\end{tabular}

Table 1: Contributions to Periodic Cruise Research

\section{Periodic Cruise Trajectory}

The term periodic cruise trajectory is almost misleading and deserves a strict definition. Technically, steady-state cruise can be considered periodic, having zero amplitude and/or zero frequency. A periodic trajectory is considered as any trajectory in which the optimal is not steady-state cruise. The determination of the non-optimality of steady-state cruise is deferred to the next section. It should also be noted that periodic trajectories do not assume perfect sinusoids. The optimal periodic trajectory is not a simple cosine wave. If this were the case, determining the optimal trajectory would be far simpler. It also is not even necessary for periodic trajectories to return to their initial state at the end of one period, as is seen in damped periodic trajectories. Periodic trajectories to date appear to be cosine in form but have extra harmonics with relatively small coefficients that can greatly affect the optimization.

The questions that arise are "Why wouldn't steady-state cruise be optimal?", "What are the fundamental principles and mechanisms that make periodic trajectories work,"?, "What is the optimal trajectory,"?, etc., etc... To answer what steady-state cruise is not optimal one could answer with the question, why should it be? There are an infinite number of possible trajectories to get from point A to point $\mathrm{B}$. There is an intuitive notion that a straight line is the shortest path between two points (in Euclidean flat space). If time were of the essence, this straight line would be the best trajectory. But the question that is more important is which trajectory takes the less amount of fuel/energy? If one were traveling at hypersonic speeds would it really matter whether the vehicle got across the ocean in 35 minutes as opposed to 30 minutes or would one rather get there in 35 minutes with a 10 percent increase in payload? In regards to steadystate cruise Gilbert has said "While this approach has considerable intuitive appeal it is not always the best. There are many examples, taken mostly from the field of chemical 
engineering, where time-dependent periodic control of the process yields improved performance."

In terms of fundamental principles and mechanisms this must be split into its two components. When engineers speak of principles, they usually refer to mass, momentum, and energy. It is the latter which is responsible for periodic trajectories to be fuel optimal over steady-state cruise. Steady-state cruise is just not good energy management. This is especially true at hypersonic speeds. One can agree that it is the goal of a vehicle to be able to fly with the most energy for as little amount of fuel. Now assume that a hypersonic vehicle has already accelerated up to speed. It is the desire of the vehicle to traverse a distance using its engine to propel itself through the air and expend fuel. Now the vehicle has certain energy. By the first law of thermodynamics it is known that this energy can change based on the work done on the vehicle and the heat added to the vehicle.

The energy of the vehicle is the summation of potential (due to the vehicle flight altitude) and kinetic (due to the vehicle speed) energies. The work done on the vehicle is in the form of drag with thrust being work done by the vehicle. The viscous aerodynamic effects are responsible for the heat added to the vehicle. This work and heat through the trajectory cause a decrease in the energy of the system. It is for this reason that thrust must be added to counter the dissipative effects of the atmosphere. Now, the question is: Can these parameters (height, velocity, thrust, drag, and heat addition) be manipulated such that the highest energy of a trajectory for the least amount of fuel is obtained? For steady-state cruise the energy is constant (if one neglects the change in mass which for short distances is negligible). However it is the integral of energy over the trajectory that is most important.

Thus, it may be possible to vary energy throughout the trajectory as long as the integral of energy is higher for a lower amount of fuel consumption. The mechanisms which one is allowed to play with is the thrust of how long the engine is allowed to burn for, the drag which is effected by the vehicle shape/angle of attack and the density of the atmosphere, and the heating done on the system, which is primarily due to drag and so is due to vehicle shape/angle of attack and the density of the atmosphere also. Thus one has many possible trajectories to get from point A to point B. Buy using kinetic energy and transferring it to potential energy a vehicle can fly at a higher altitude were the atmosphere is thinner thus producing less drag and heating.

Since there will always be some loss due to drag and heating, thrust will have to be used to make up for this. Periodic trajectories do this in the most efficient manner. Thus, they maximize the amount of energy over a trajectory for a given amount of fuel. Now it should be noted that periodic trajectories may not always be best due to a given vehicle configuration and handling profile. Obviously the vehicle will have to change its heading and angle of attack in order to increase its altitude. Certain shapes may not have desirable off design aerodynamic profiles so that the drag encountered by flying off design is greater than that saved by flying higher.

The question of what the optimal trajectory looks like cannot be answered. Every case is different. It is necessary to determine the optimal manner in which to get the best energy efficiency for a given amount of fuel. Every vehicle will have different properties that will effect what the optimal trajectory will look like. Optimal control theory cannot tell you in an analytical sense what the best trajectory is only whether a candidate 
trajectory is optimal. Optimization techniques must be used to determine the vest trajectory. The next section will detail the methods available for determining whether a trajectory is optimal or not and how to obtain the optimal.

\section{Mathematical Analysis: Non-optimality of Steady Cruise}

While the previous sections have provided a historical and an intuitive perspective of why periodic cruise trajectories are fuel-optimal, this section attempts to present a mathematical argument illustrating why steady cruise trajectories are non-optimal for minimum fuel solutions. Ideally, one would like to mathematically argue that periodic cruise is fuel-optimal. This analysis, however, is beyond the scope of the current work. Nevertheless, it is equally important to discuss why steady cruise may be non-optimal.

The objective of minimum fuel trajectories is to find a trajectory that minimizes some scalar cost function J, given generically by:

$$
J(\mathbf{u})=h\left(\mathbf{x}\left(t_{f}\right), t_{f}\right)+\int_{t_{o}}^{t_{f}} g(\mathbf{x}(t), \mathbf{u}(t), t) d t
$$

Applying the calculus of variations to this cost function or the Hamiltonian of this cost function, requires that in order for the existence of a local minimum that the first and second derivatives must satisfy the following necessary and sufficient conditions:

$$
\frac{\delta \mathbf{f}}{\delta \mathbf{x}}=\left[\begin{array}{cccc}
\frac{\delta f_{1}}{\delta x_{1}} & \frac{\delta f_{1}}{\delta x_{2}} & \cdots & \frac{\delta f_{1}}{\delta x_{n}} \\
\cdot & \cdot \\
\cdot & \cdot \\
\frac{\delta f_{k}}{\delta x_{1}} & \frac{\delta f_{k}}{\delta x_{2}} & \cdots & \frac{\delta f_{k}}{\delta x_{n}}
\end{array}\right]=0
$$

With the above matrix being called the Jacobian, and:

$$
\frac{\delta^{2} \mathbf{f}}{\delta \mathbf{x}^{2}}=\left[\begin{array}{cccc}
\frac{\delta^{2} f_{1}}{\delta x_{1}^{2}} & \frac{\delta^{2} f_{1}}{\delta x_{2}{ }^{2}} & \cdots & \frac{\delta^{2} f_{1}}{\delta x_{n}{ }^{2}} \\
\cdot & \cdot \\
\cdot & \cdot \\
\frac{\delta^{2} f_{k}}{\delta x_{1}^{2}} & \frac{\delta^{2} f_{k}}{\delta x_{2}{ }^{2}} & \cdots & \frac{\delta^{2} f_{k}}{\delta x_{n}^{2}}
\end{array}\right]=0
$$

Lets define the Hamiltonian as: 


$$
H(\mathbf{x}(t), \mathbf{u}(t), \mathbf{p}(t), t) \equiv g(\mathbf{x}(t), \mathbf{u}(t), t)+\mathbf{p}^{T}(t)[\mathbf{a}(\mathbf{x}(t), \mathbf{u}(t), t)]
$$

Where $\mathrm{p}$ is the co-state vector, also known as Lagrange multipliers. Using this notation the necessary conditions for optimality are:

$$
\begin{aligned}
& \dot{\mathbf{x}}^{*}(t)=\frac{\delta H}{\delta \mathbf{p}}\left(\mathbf{x}^{*}(t), \mathbf{u}^{*}(t), \mathbf{p}^{*}(t), t\right) \\
& \dot{\mathbf{p}}^{*}(t)=\frac{\delta H}{\delta \mathbf{p}}\left(\mathbf{x}^{*}(t), \mathbf{u}^{*}(t), \mathbf{p}^{*}(t), t\right) \\
& 0=\frac{\delta H}{\delta \mathbf{u}}\left(\mathbf{x}^{*}(t), \mathbf{u}^{*}(t), \mathbf{p}^{*}(t), t\right)
\end{aligned}
$$

(where the above equations must hold for all $t \in t_{\mathrm{o}}, \mathrm{t}_{\mathrm{f}}$ ), and

$$
\left[\frac{\delta h}{\delta \mathbf{x}}\left(\mathbf{x}^{*}\left(t_{f}\right), t_{f}\right)-\mathbf{p}^{*}\left(t_{f}\right)\right]^{T} \delta \mathbf{x}_{\mathbf{f}}+\left[H\left(\mathbf{x}^{*}\left(t_{f}\right), \mathbf{u}^{*}\left(t_{f}\right), \mathbf{p}^{*}\left(t_{f}\right), t_{f}\right)+\frac{\delta h}{\delta t}\left(\mathbf{x}^{*}\left(t_{f}\right), t_{f}\right)\right] \delta t_{f}=0
$$

These are first order necessary conditions for optimality. However, first order optimality conditions are not sufficient to determine if a solution is a minimum. Second order tests are needed to fully determine the optimality of a particular trajectory. The second derivative of the Hamiltonian with respect to the control variables must be nonnegative:

$$
\frac{\delta^{2} H}{\delta u^{2}}\left(\mathbf{x}^{*}(t), \mathbf{u}^{*}(t), \mathbf{p}^{*}(t), t\right)>0
$$

These necessary and sufficient conditions can be applied to the steady-state cruise cost function given by Speyer for a vehicle flying steady state cruise trajectories. This cost has the following form:

$$
J=\int_{0}^{t_{f}} \sigma T d t
$$

With the corresponding Hamiltonian based on flight dynamics of an aircraft in a plane given by:

$$
H=\sigma T+\lambda_{V}\left(\frac{T-D}{M}-g \sin \gamma\right)+\lambda_{\gamma}\left(\frac{L-W \cos \gamma}{M V}\right)+\lambda_{h}(V \sin \gamma)+\lambda_{x}(V \cos \gamma)
$$


It is not difficult to show that the first order necessary conditions are satisfied along the steady cruise arc with the Lagrange multipliers, or co-states, satisfying:

$$
\begin{aligned}
& \lambda_{V}=-\sigma M \\
& \lambda_{x}=-\frac{\sigma D}{V} \\
& \lambda_{\gamma}=-\sigma D V M \\
& \lambda_{h}=-\frac{\sigma W}{V}
\end{aligned}
$$

Thus, the cruise arc does in fact satisfy the stationary conditions for optimality. The next step, albeit more complicated, is to determine whether the $2^{\text {nd }}$ order conditions for a minimum are satisfied for the steady-state cruise trajectory. Essentially, Speyer linearizes the equations of motion to generate a state space model of the system dynamics about a cruise fight condition. This leads to the following state space representation:

$$
\dot{x}=A x+B y
$$

Where:

$$
\begin{aligned}
& x^{T}=[\delta V, \delta \gamma, \delta h, \delta x] \\
& y^{T}=[\delta T, \delta L]
\end{aligned}
$$

Where matrices A and B contain the linear flight dynamics about a steady cruise arc. Using this form of the dynamics and the following accessory cost function:

$2 \delta^{2} J=\int_{t_{o}}^{t_{f}}\left[x_{T} y_{T}\right]\left[\begin{array}{ll}Q & C \\ C & Q\end{array}\right]\left[\begin{array}{l}x \\ y\end{array}\right] d t$

the $2^{\text {nd }}$ order sufficient condition for optimality involves determining whether the frequency response matrix:

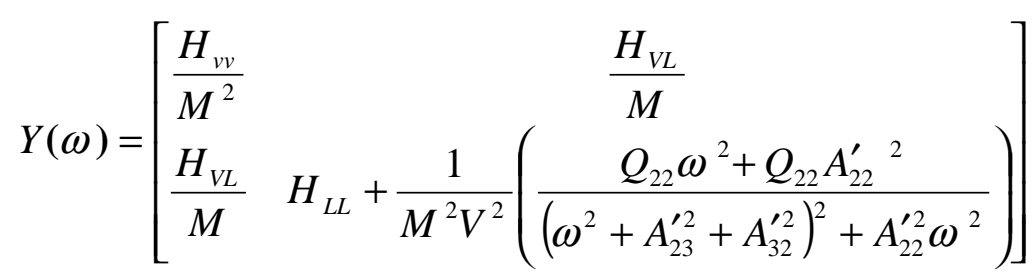

Remains non-negative over the entire frequency range of interest. This matrix reduces to the following scalar form 
$Y(\omega)=\frac{K}{H_{V V}}+\frac{1}{M^{2} V^{2}}\left(\frac{Q_{22} \omega^{2}+Q_{22} A_{22}^{\prime}{ }^{2}}{\left(\omega^{2}+A_{23}^{\prime 2}+A_{32}^{\prime 2}\right)^{2}+A_{22}^{\prime 2} \omega^{2}}\right)$

This function must remain non-negative for all $\bullet$ Speyer illustrates that for typical aerodynamic Drag models of conventional aircraft that steady-state cruise is not minimizing over all frequencies. This implies that the $2^{\text {nd }}$ order condition for optimality is violated. Thus, steady cruise cannot be fuel-optimal suggesting that other trajectories such as periodic may be more fuel optimal.

\section{POST COMPUTATIONAL SIMULATION}

The previous sections have attempted to provide some insights as to why periodic cruise trajectories may be fuel optimal. This section attempts to computationally simulate periodic cruise trajectories using NASA's POST (Program to Optimize Simulated Trajectories) software code for hypersonic flight. POST was developed to primarily fly ascent profiles of rocket-based launch vehicles including the space shuttle. In addition, it has also been modified to handle low-earth orbiting satellites. However, it is not ideally suited to handle hypersonic flight trajectories because of the lack of aerodynamic and propulsion input data decks for integrated hypersonic vehicle designs. Nevertheless, an attempt was made to simulate periodic cruise flight of a hypersonic vehicle using models of ramjet and scramjet engines. Aerodynamic models were derived from previous hypersonic vehicle designs. Simulated results are obtained displaying the altitude profile, Mach number, dynamic pressure, angle of attack and $g$ loading. These results indicate that it is possible to simulate periodic trajectories using POST. However, fuel-saving results from these ad-hoc simulations are inconclusive since the periodic nature of the trajectory was achieved by turning the engine on at arbitrary times that were not necessarily optimal. The most important aspect of the simulation results in Figures 2 thru 17 is that POST can be configured to handle hypersonic flight trajectories, albeit with a lot of effort to generate appropriate input data decks from integrated vehicle designs. 


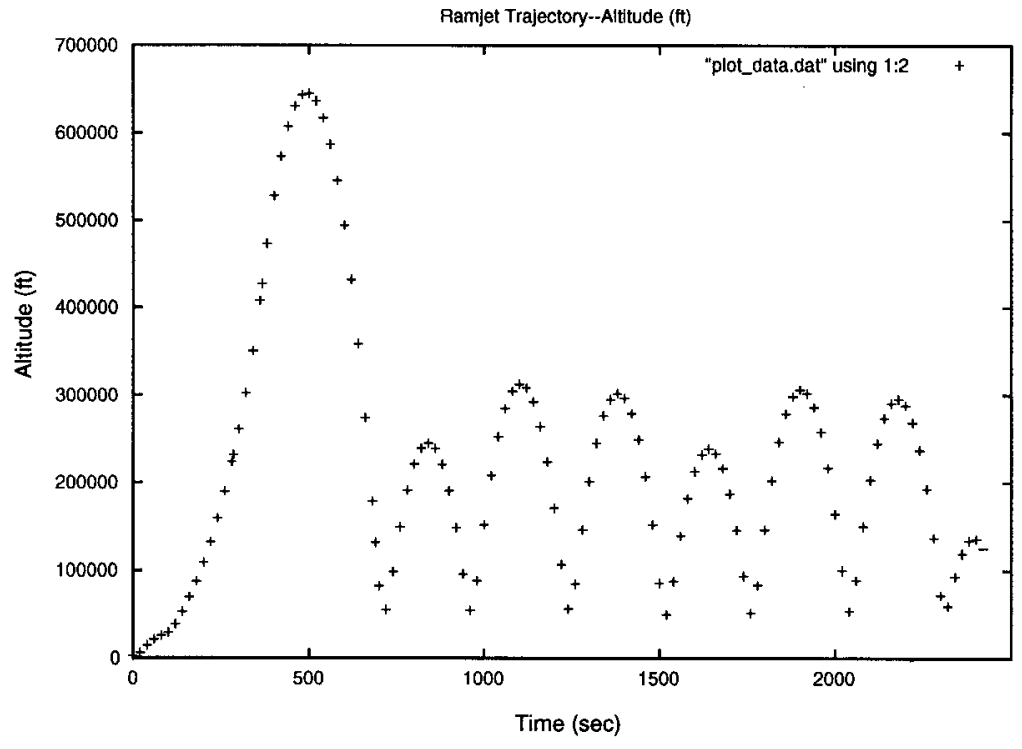

Figure 2: Ramjet Altitude Profile 


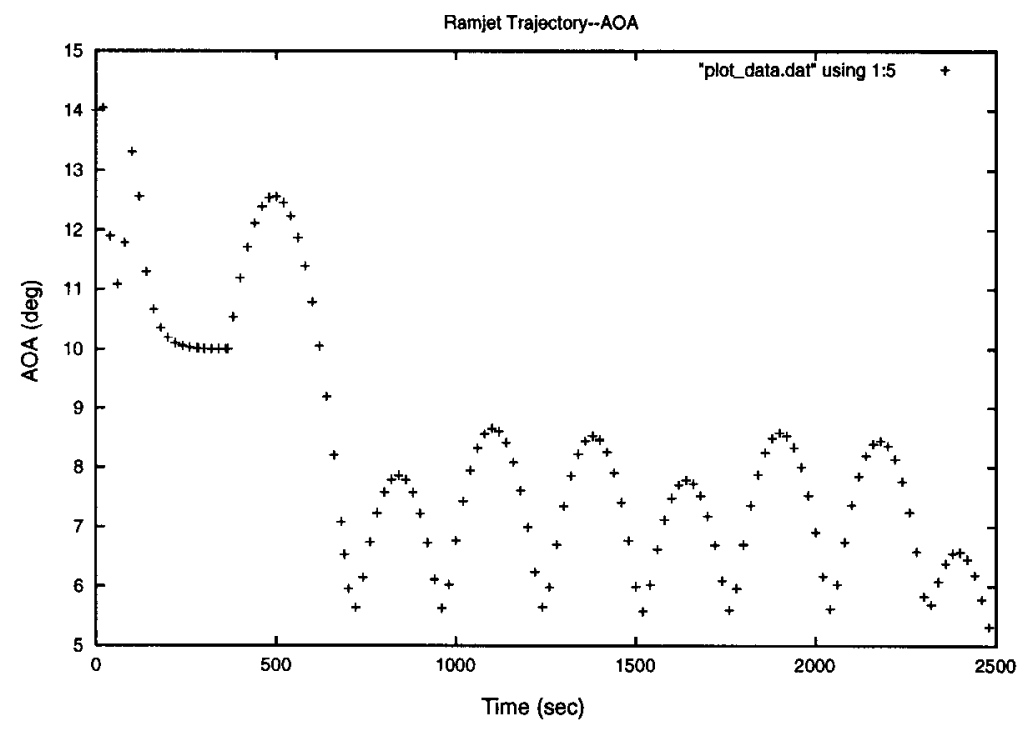

Figure 3: Ramjet AOA Profile

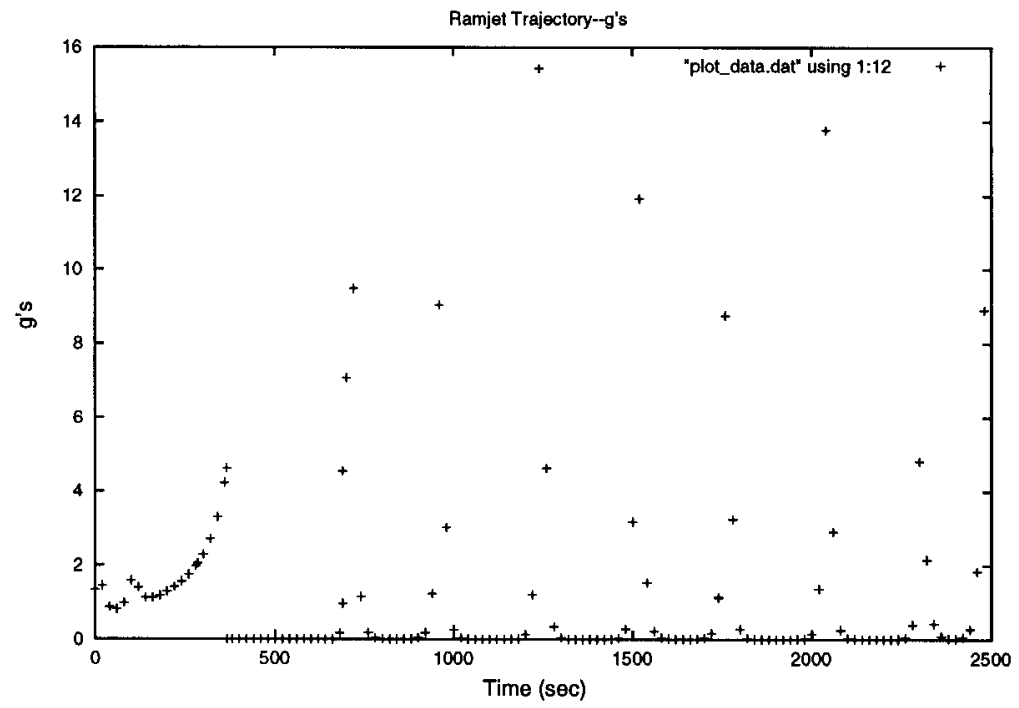

Figure 4: Ramjet G's Profile 


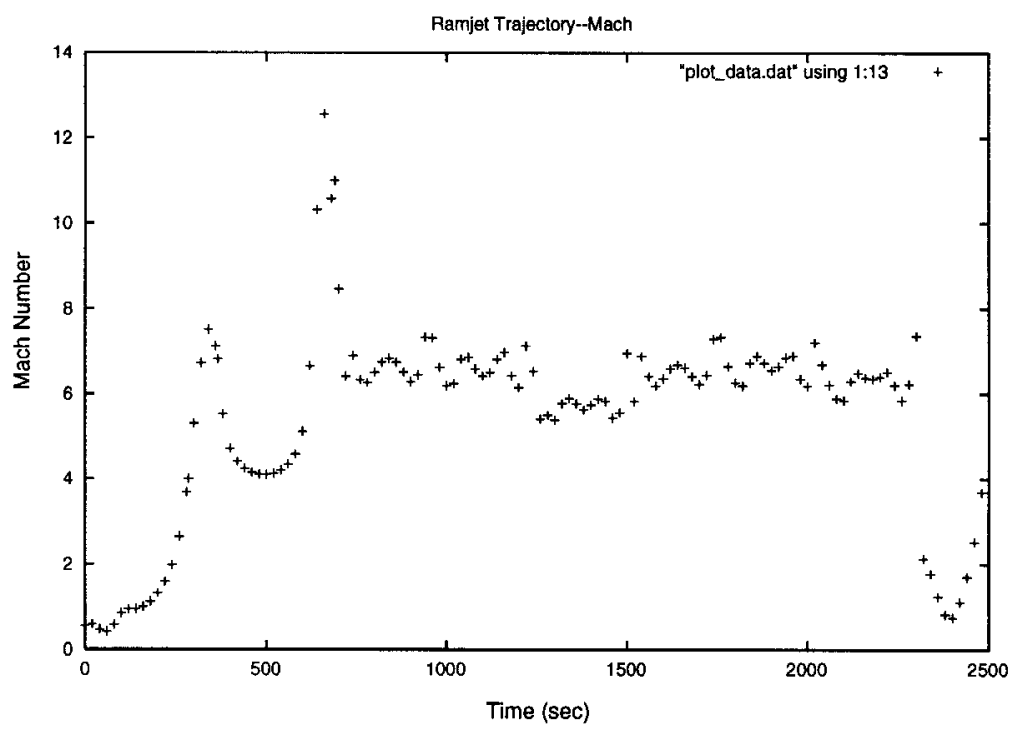

Figure 5: Ramjet Mach Profile

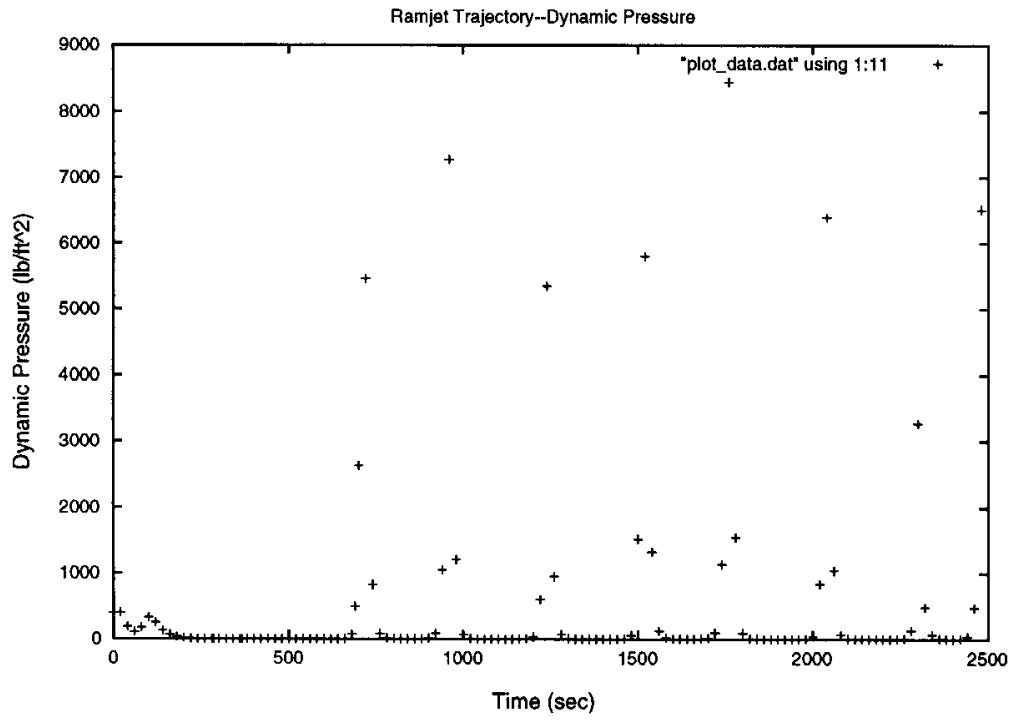

Figure 6: Ramjet Dynamic Pressure Profile 


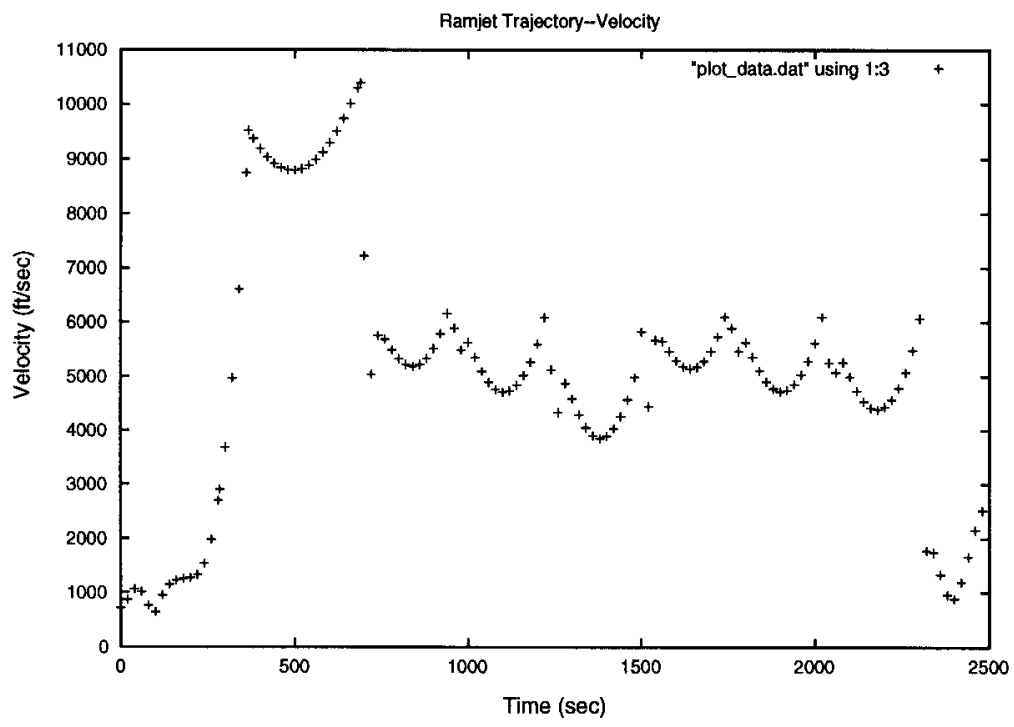

Figure 7: Ramjet Velocity Profile

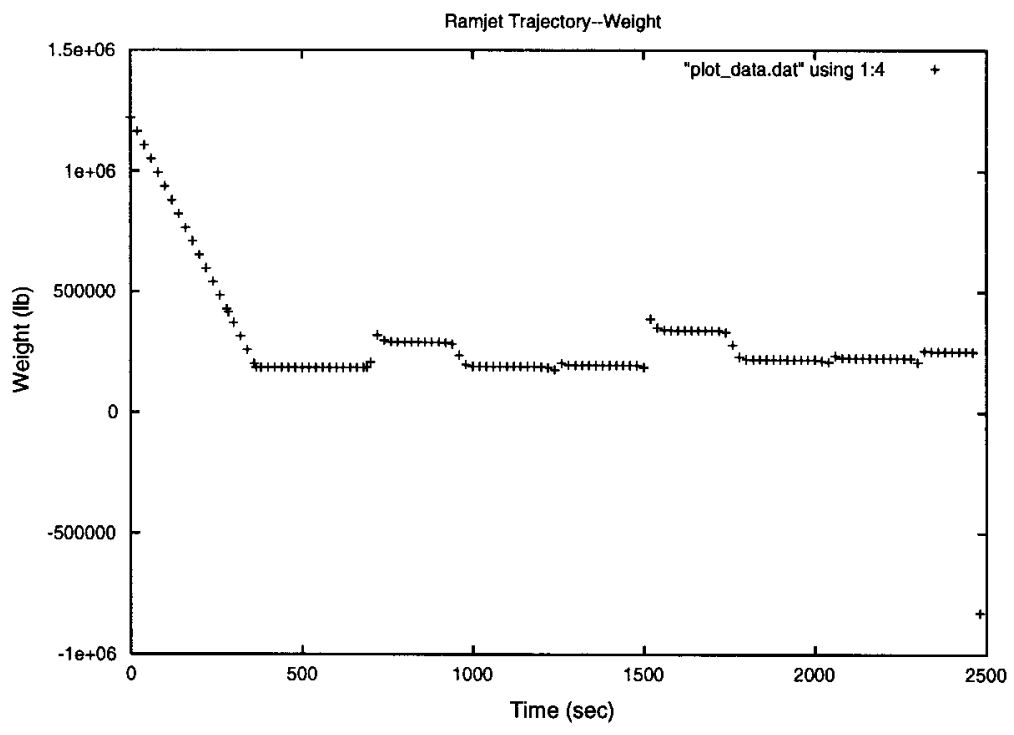

Figure 8: Ramjet Weight Profile 


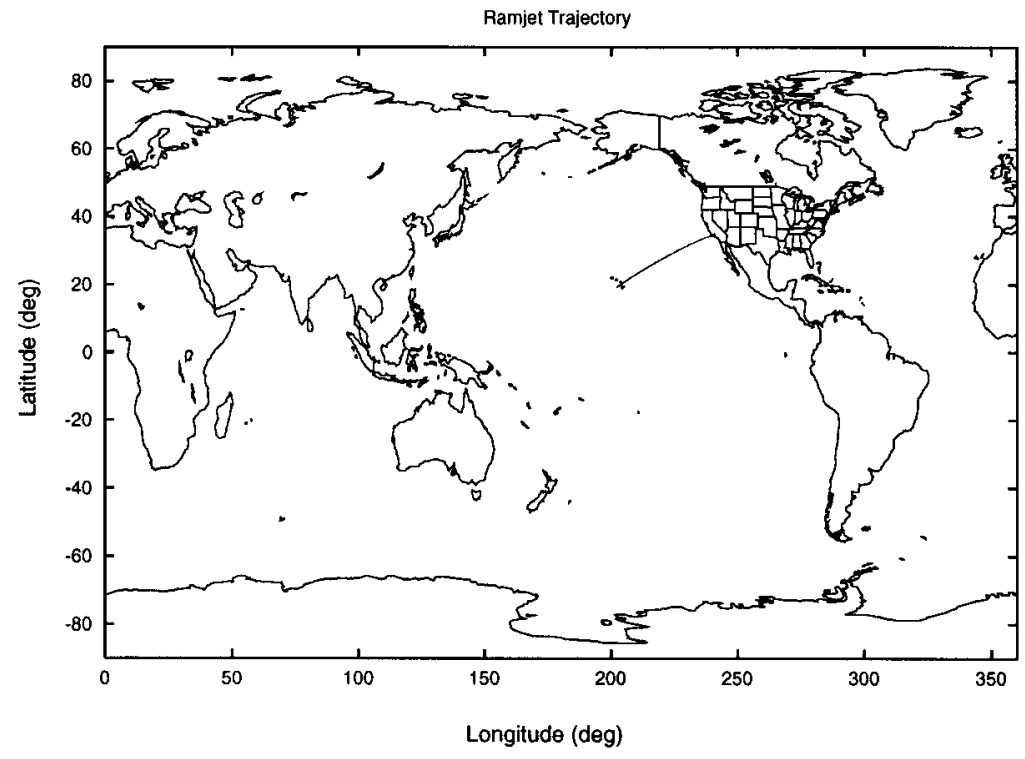

Figure 9: Ramjet World Map Profile

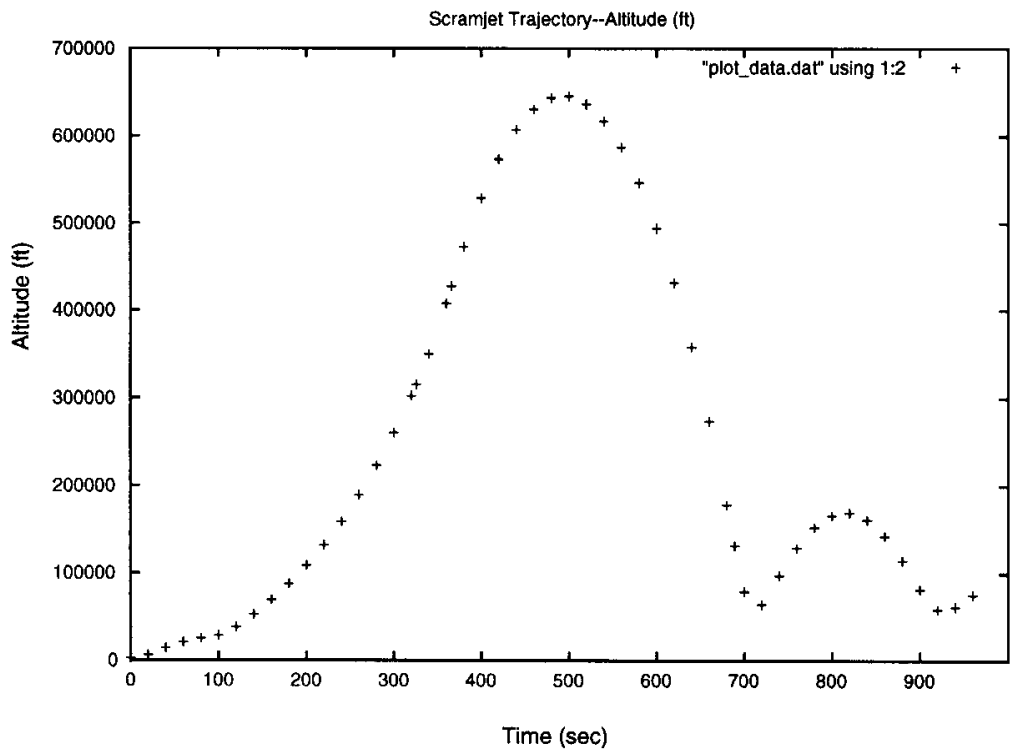

Figure 10: Scramjet Altitude Profile 


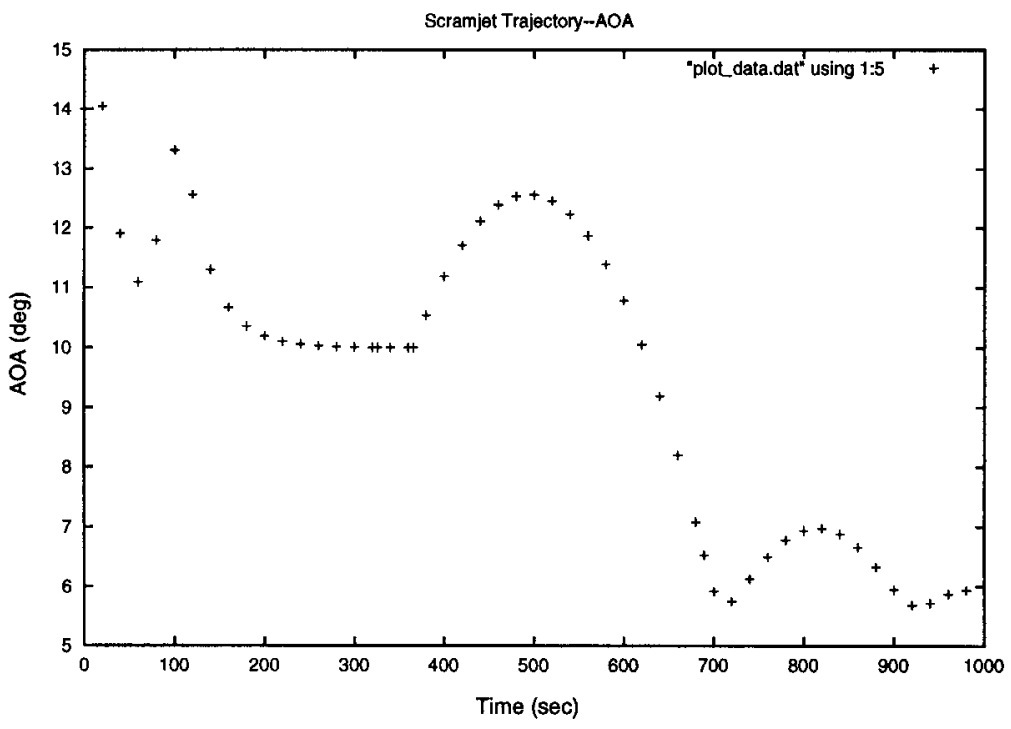

Figure 11: Scramjet AOA Profile

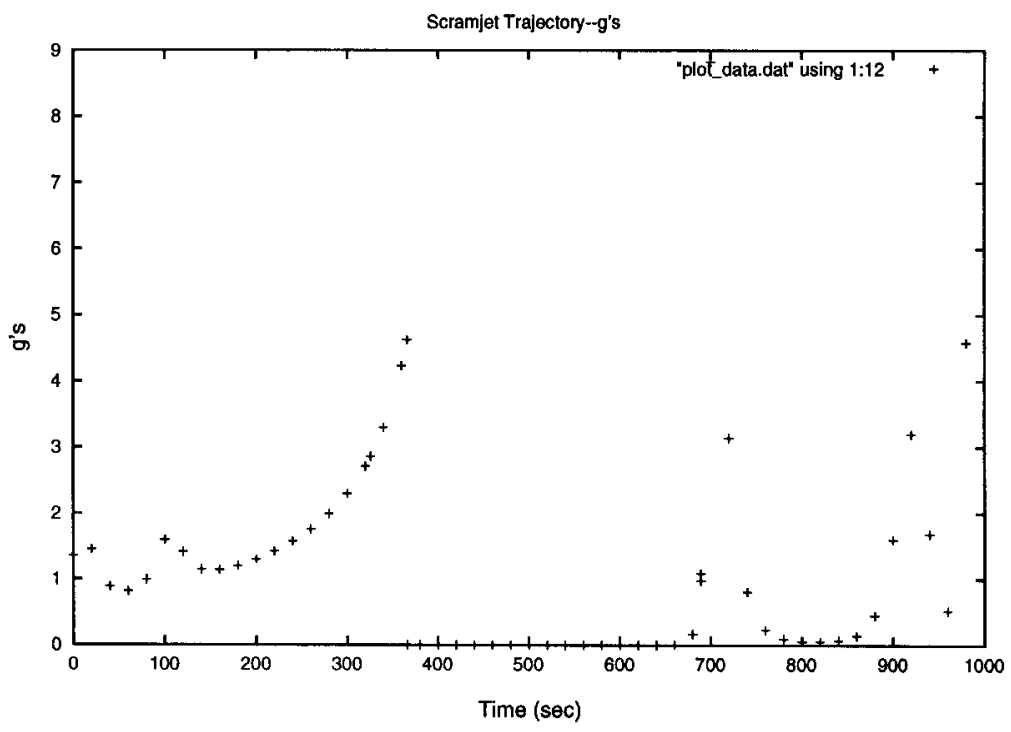

Figure 12: Scramjet G Profile 


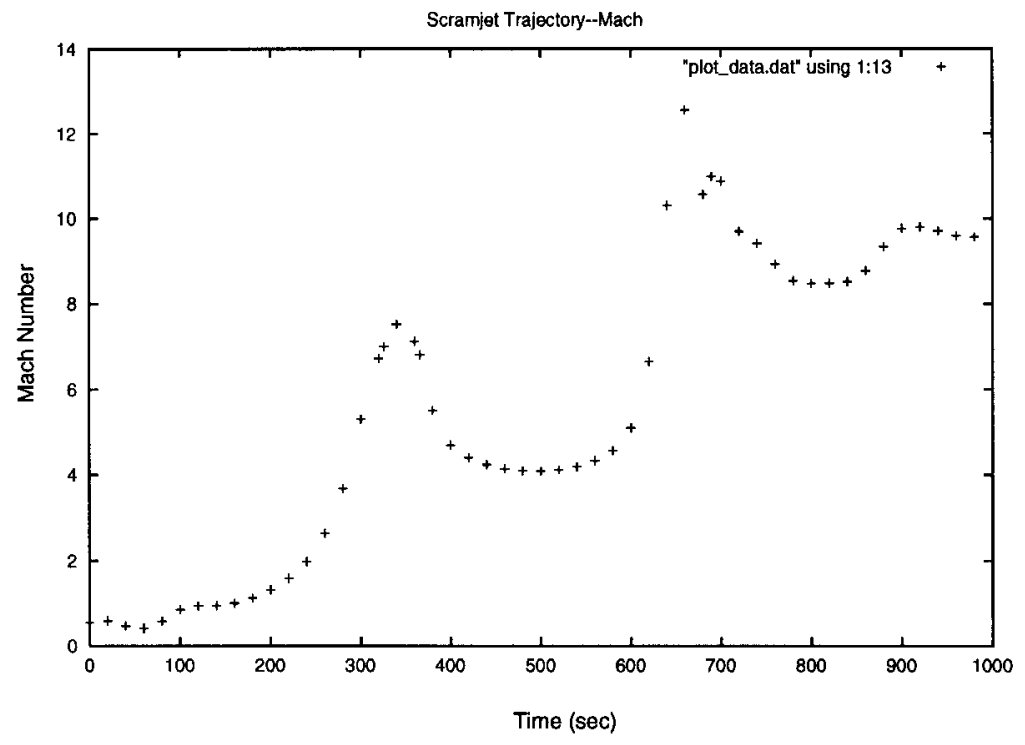

Figure 13: Scramjet Mach Profile

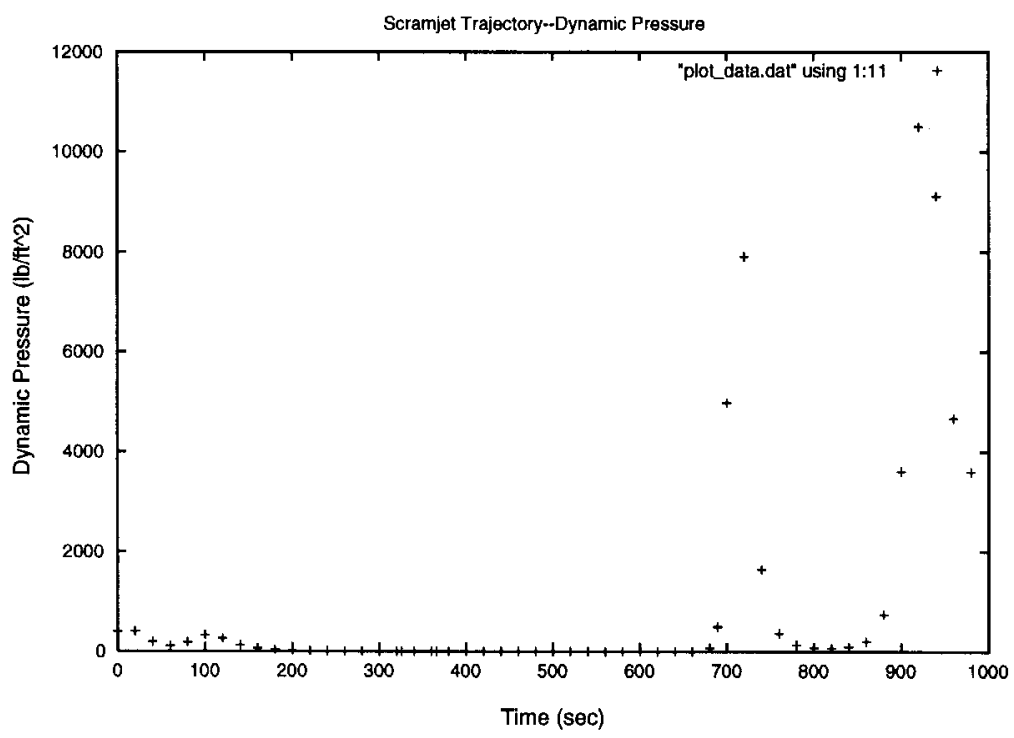

Figure 14: Scramjet Dynamic Pressure Profile 


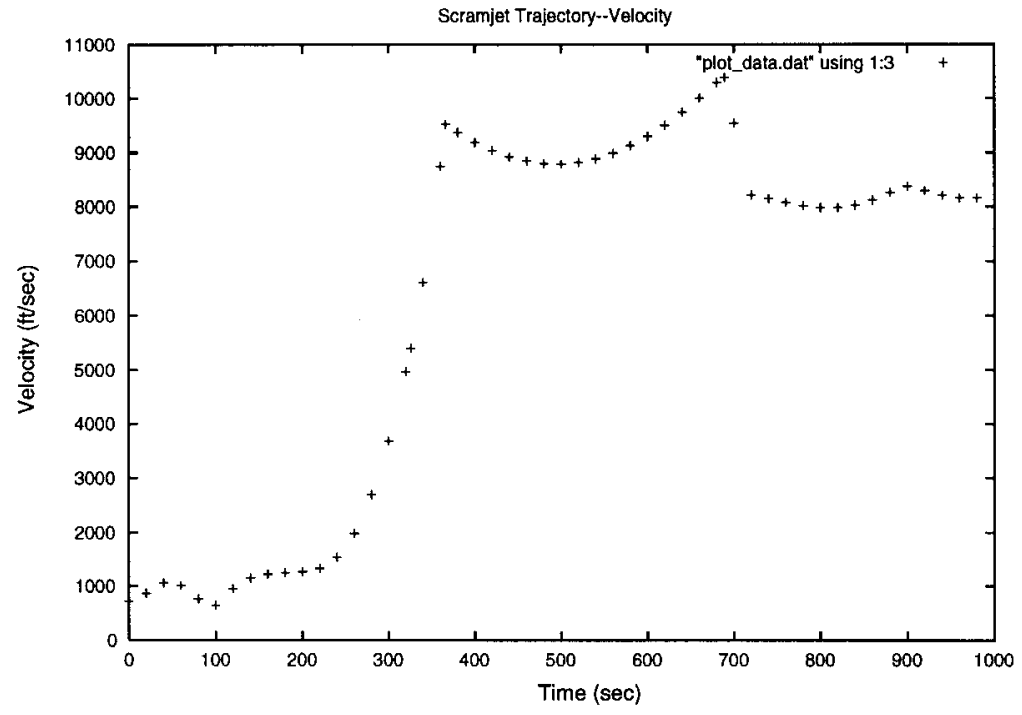

Figure 15: Scramjet Velocity Profile

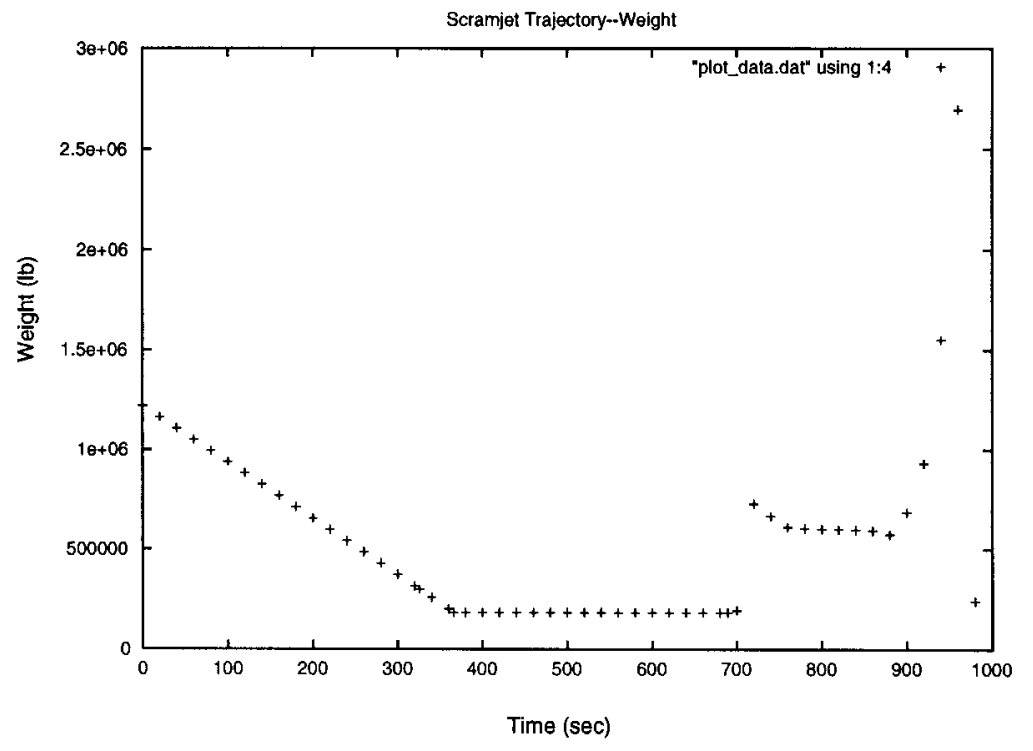

Figure 16: Scramjet Weight Profile 


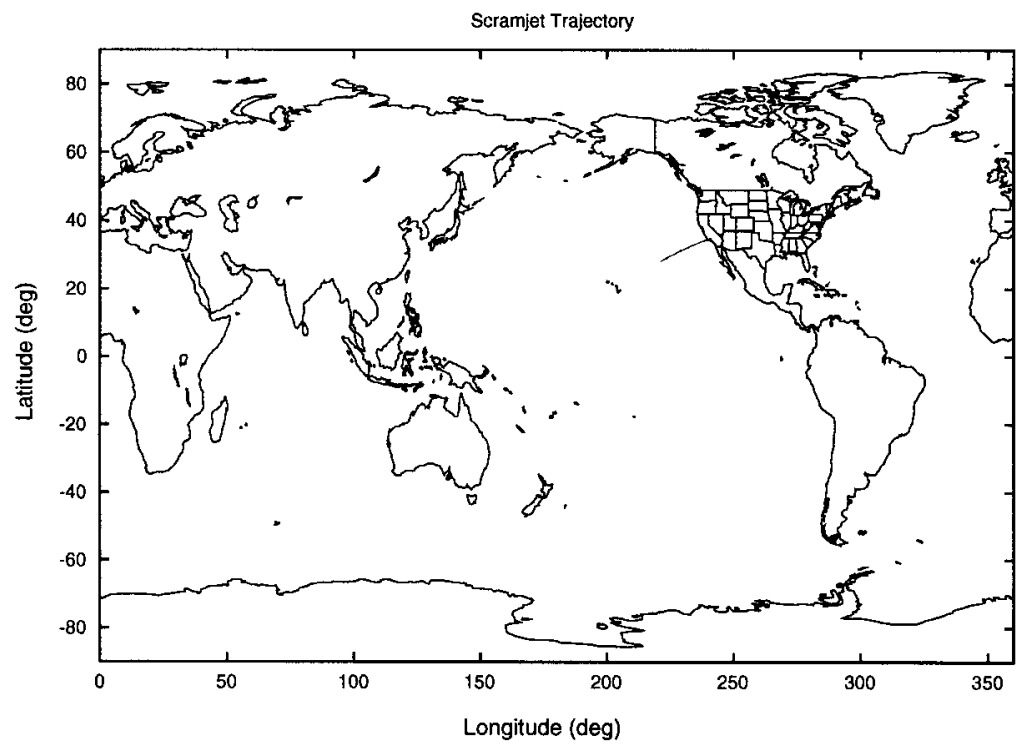

Figure 17: Scramjet World Map Profile

\section{Vehicle Design}

The concept "HyperSoar" is still very new. Most of the effort to date has been in proving or disproving the advantages of HyperSoar's periodic trajectory. Of course, a notional vehicle design is required to support these trajectory studies and to help describe the overall concept. This current work advanced and refined the vehicle design. Although the vehicle design can still be called "notional," its refinement is helping other researches concentrate their work.

Previous notional vehicle designs of a HyperSoar vehicle were based upon cone derived waveriders suggested by Rasmussen ${ }^{22}$. It was known at the time that this vehicle configuration did not address aerodynamic issues concerning base drag and did not adequately address flight control. In the course of this work, the notional design of the vehicle was improved using Osculating Cone waverider design ${ }^{23}$. Figure 18 shows the layout of this new design. The inlet airflow requirements of the propulsion are more adequately addressed in this design. Also the aft-body shape and flight control layout are more realistic and provide a better starting point for more detailed vehicle design. A great deal of vehicle design effort is required, but was beyond the scope of this current study. NASA Ames Research Center and the University of Maryland Aerospace Engineering department have begun internal studies of the aerodynamic design of this new notional vehicle design.

${ }^{22}$ Rasmussen, M. L., "Analysis of Cone-Derived Waveriders by Hypersonic Small-Disturbance Theory," in Proceedings of the $1^{\text {st }}$ International Hypersonic Waverider Symposium, October 1990.

23 Takashima, N., Lewis M. J., "Optimization of Waverider Based Hypersonic Cruise Vehicles with OffDesign Considerations," Journal of Aircraft, Vol. 36, No. 1, pp. 235-245, Jan.-Feb. 1999. 


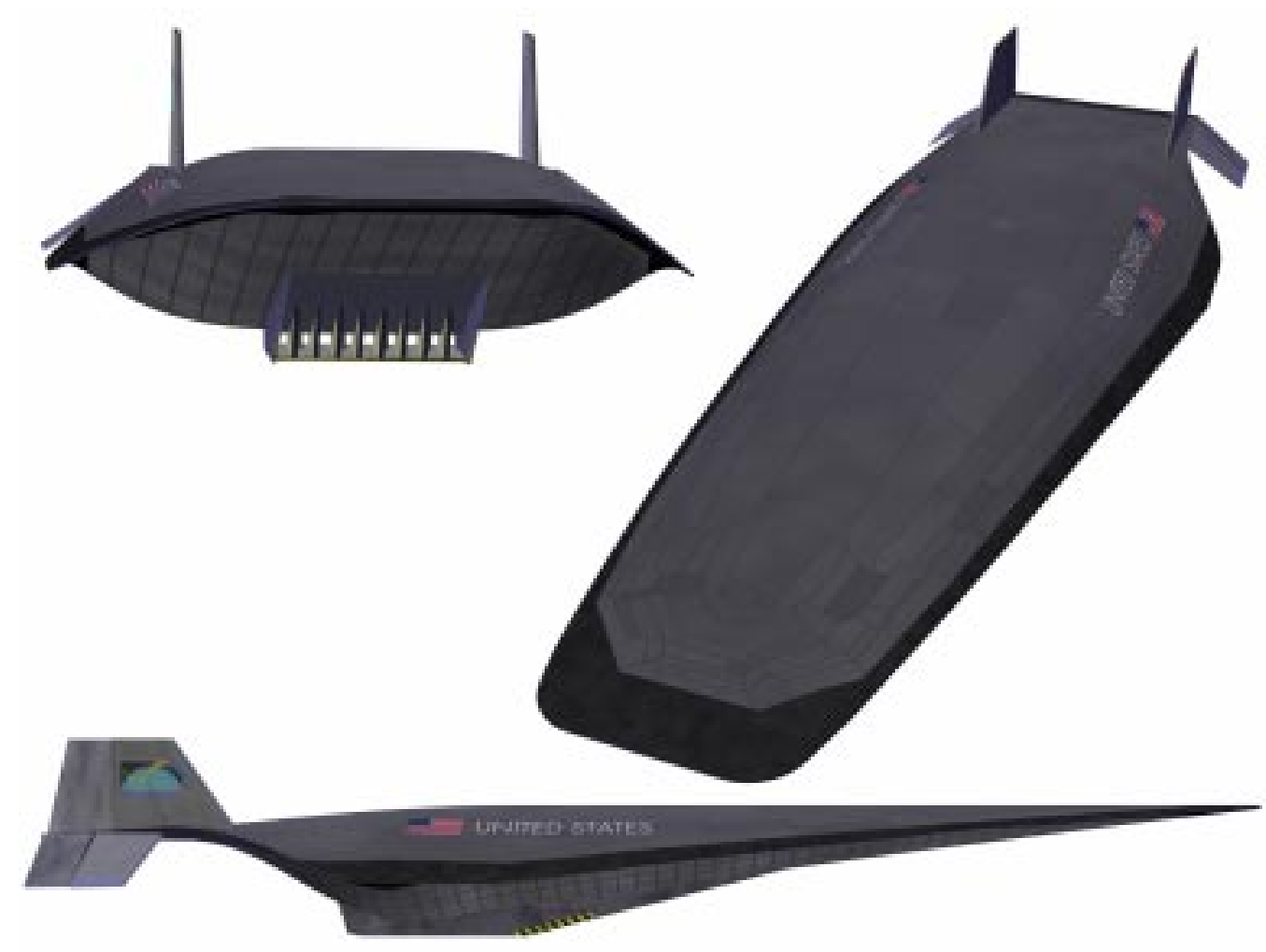

Figure 18: HyperSoar Vehicle Layout Based Upon Osculating Cone Waverider Design

The authors had prior knowledge that Rocket Based Combined (RBCC) engines have many of the attributes desired in the propulsion for HyperSoar. The most important requirement being that an engine must be able to operation over the entire velocity range of HyperSoar's flight envelope. Using conventional propulsion technology, this would require multiple engines, since conventional engines (turbojet, -fan, ramjet, rocket) can operate over only limited ranges of velocity. In a single engine, RBCC engines can operate over the entire flight envelope. This promises to reduce complexity and save engine mass. In addition, HyperSoar requires the engine to operate as an accelerator engine at all times. That is, even in cruise, HyperSoar's engines are use only to accelerate the vehicle. Billig ${ }^{24}$ showed that $\mathrm{RBCC}$ engines have superior accelerator performance over other engine types.

RBCC engines are still a new technology and the first examples of these engines are currently under test ${ }^{25}$. For HyperSoar studies, engineering models of the performance and weight of representative RBCC engines are required. Aerojet, one of the developers of a RBCC engine, gave us a spreadsheet model of their RBCC engine. In addition a

$24 \quad$ Billig, F. S., "The Integration of the Rocket with the Ram-Scramjet as a Viable Transatmospheric Accelerator," AL-94-P268, John Hopkins University Applied Physics Laboratory, 1994.

25 http://stp.msfc.nasa.gov/astp/rbcc.html 
generic RBCC engine model was obtained from Georgia Institute of Technology ${ }^{26}$. These models will be implemented into future design studies.

\section{SUMMARY}

This report has provided some preliminary insights as to why periodic hypersonic cruise trajectories are possibly fuel optimal. The periodic cruise arc attempts to strike a balance between aerodynamic and propulsion efficiency of a high-speed vehicle design at altitude. This involves an exchange between kinetic and potential energy to ensure that the total energy of the vehicle remains approximately constant over a cycle. It appears that based on this exchange of kinetic and potential energy that steady cruise trajectories do not provide for an optimal balance. In fact Speyer has shown mathematically that there are times when steady cruise trajectories violate the $2^{\text {nd }}$ order sufficient conditions for the existence of a local minimum. While a similar proof does not exist currently for periodic cruise trajectories, it does rule out steady cruise as a fuel-optimal solution. Computationally periodic hypersonic cruise trajectories can be simulated, but appropriate aerodynamic and propulsion data decks must be developed to serve as input to the POST simulation code. Overall the POST code is difficult to work with since it has not been configured for air breathing propulsion with high speed vehicle designs. Thus, additional work is required to develop the necessary software interfaces that enable appropriate use of the POST code for determining optimal periodic hypersonic cruise arcs. This is an important phase of this work since many government and industry officials have confidence in results that are derived using POST. This is primarily because the code has been validated for many aerospace vehicles. In addition, developing the mathematical analysis to prove or disprove the fuel-optimality of periodic cruise arcs would be extremely valuable to support any computational results.

${ }^{26}$ Olds, J, Bradford, J., "SCCREAM (Simulated Combined-Cycle Rocket Engine Analysis Module): A Conceptual RBCC Engine Design Tool," AIAA 97-2760, $33^{\text {rd }}$ AIAA/ASME/SAE/ASEE Joint Propulsion Conference, 1997. 